\title{
Right or left? Side selection for a totally implantable vascular access device: a randomised observational study
}

\author{
Wen-Ying Lin ${ }^{1,2}$, Chih-Peng Lin ${ }^{1,3}$, Chih-Hung Hsu ${ }^{3,4}$, Ying-Hui Lee ${ }^{3}$, Yi-Ting Lin ${ }^{3}$, Meng-Chi Hsu ${ }^{3}$ \\ and Yu-Yun Shao*,2,3,4 \\ ${ }^{1}$ Department of Anesthesiology, National Taiwan University Hospital, 7, Chung-Shan S Rd, Taipei City 10002, Taiwan; \\ ${ }^{2}$ National Taiwan University Cancer Center, National Taiwan University College of Medicine, 1, Sec. 1, Ren'ai Rd, Taipei \\ City 10051, Taiwan; ${ }^{3}$ Department of Oncology, National Taiwan University Hospital, 7, Chung-Shan S Rd, Taipei City 10002, \\ Taiwan and ${ }^{4}$ Graduate Institute of Oncology, National Taiwan University College of Medicine, 1, Sec. 1, Ren'ai Rd, Taipei \\ City 10051, Taiwan
}

Background: Totally implantable vascular access device (TIVAD)-related complications interfere in the anticancer treatment and increase medical expenses. We examined whether the implantation side of central line TIVADs is associated with the occurrence of thrombotic or occlusion events.

Methods: We enrolled patients with cancer who required central line TIVADs and randomised them to receive the TIVAD implantation on either the left or right side. The primary endpoint was the occurrence of catheter-related thrombotic or occlusion events.

Results: We randomised 240 patients, of which 235 received TIVAD implantation according to the protocol. In the per-protocol cohort, 117 and 118 patients received implantation on the left and right sides, respectively. Catheter-related thrombotic or occlusion events occurred in 9 (4\%) patients, accounting for 0.065 events per 1000 catheter-days. Between the patients with leftand right-sided implantations, the occurrence rates $(P=0.333)$ and the time from catheter implantation to the occurrence of thrombotic or occlusion events $(P=0.328)$ were both similar. In the multivariate analysis, the side of implantation remained unassociated with the occurrence of thrombotic or occlusion events.

Conclusions: The side of central line TIVAD implantation was not associated with the occurrence of catheter-related thrombotic or occlusion events in patients with cancer.

A central line totally implantable vascular access device (TIVAD) is commonly used in patients with cancer. A TIVAD provides stable, central line access for intravenous chemotherapy, preventing the thrombophlebitis that is common in peripheral veins. In addition, a central line TIVAD facilitates the continuous infusion of some chemotherapeutic agents such as 5-fluorouracil. For patients with advanced or terminal diseases, this device provides vascular access for high-concentration nutritional support.
TIVADs may result in some complications. Intraluminal thrombosis can occur at a frequency of 0.6-0.81 events per 1000 catheter-days (Ray et al, 1996; Schwarz et al, 2000). Manifestations include failure to draw blood from the port or failure of infusion. These events may be reversed using urokinase, streptokinase, or tissue plasminogen activators (Hurtubise et al, 1980; Doizaki and Weber, 1986; Fraschini et al, 1987). Catheter-related venous thrombosis is more severe and can cause swelling of the arm, neck,

*Correspondence: Dr Y-Y Shao; E-mail: yuyunshao@gmail.com

Received 19 April 2017; revised 11 June 2017; accepted 17 July 2017; published online 8 August 2017

(C) 2017 Cancer Research UK. All rights reserved 0007-0920/17 
and head and erythema of the extremities. Five to 41 per cent of patients developed such symptomatic thrombi (Kuter, 2004). Catheter infection is also common and highly associated with intraluminal thrombosis and catheter-related venous thrombosis (Press et al, 1984; Barzaghi et al, 1995; Eastman et al, 2001). All of these complications may delay the chemotherapy course and even be life threatening.

Several factors have been associated with catheter-related thrombotic or occlusion events (intraluminal thrombosis or catheter-related venous thrombosis), such as cancer types, catheter types, and the location of the catheter tips (Stanislav et al, 1987; Anderson et al, 1989; Eastridge and Lefor, 1995; Craft et al, 1996; De Cicco et al, 1997; Nightingale et al, 1997; Schwarz et al, 2000; Kuter, 2004; Shivakumar et al, 2009). Previous studies also reported that compared with catheters inserted on the right side, those inserted on the left side resulted in more catheter-related thrombotic events (Gould et al, 1993; Craft et al, 1996; Shivakumar et al, 2009; Murray et al, 2013). However, no randomised studies have yet investigated the difference between TIVADs inserted on the left and right sides. Because all previous studies were nonrandomised observational or retrospective analyses, their results could be confounded by other risk factors. Therefore, we conducted a randomised observational study to investigate whether the side of central line TIVAD implantation was associated with the occurrence of thrombotic or occlusion events in patients with cancer.

\section{PATIENTS AND METHODS}

Study design. This randomised observational study was conducted at National Taiwan University Hospital (NTUH), Taipei, Taiwan. After obtaining informed consent from all patients, they were randomly assigned an implantation side. Randomisation was performed by investigators with no access to the patients by using a free randomisation software developed by $\mathrm{Dr}$ M. Saghaei from Isfahan University of Medical Sciences in Iran (http://mahmoodsaghaei.tripod.com/Softwares/randalloc.html). Lung cancer and ovarian cancer were used as stratification factors. According to the standard procedures, the patients received central line TIVAD implantation surgery. This study was approved by the Research Ethic Committee of NTUH and registered at www.clinicaltrials.gov (NCT01525277).

Patient eligibility. Patients were eligible for inclusion if they were aged 20 years or older, had received a diagnosis of cancer, and required central line TIVAD implantation. Patients who had breast cancer, mediastinal tumours exceeding $6 \mathrm{~cm}$ in diameter, an Eastern Cooperative Oncology Group performance status of $>2$, difficulty in receiving standing posteroanterior view chest X-ray examination, or a prior history of TIVAD implantation were excluded. In addition, patients with conditions preventing the randomisation of the implantation side were excluded.

Surgical procedures. Within 7 days before surgery, patients' haemogram, coagulation time, and blood levels of D-dimer and fibrinogen were examined as routine clinical practice at the NTUH laboratory. We followed a previously reported surgical procedure for TIVAD implantation (Lin et al, 2011). Briefly, all patients were sedated, with spontaneous ventilation maintained during the procedure. The longitudinal axis of the axillary vein was identified using ultrasound. The ultrasound probe was held perpendicular to the skin, and the skin was marked for site preparation. The long axis of the axillary vein was assessed by $2 \mathrm{D}$ image and confirmed by pulse wave Doppler. Needle insertion trajectory was also determined. In this scanning plane, we made certain there were no overlapping artery or brachial plexus neural tissues. We used an 18-gauge intravenous catheter for venipuncture. The standard
Seldinger technique with a peel-away introducer sheath was used to introduce the guide wire and connect to a 7.8-F polyurethane catheter (Port-A-Cath; SIMS Deltec, Inc., St Paul, MN, USA). The position of the catheter tip was confirmed using chest X-ray films obtained immediately after the completion of the procedure in the post-anaesthesia care unit. All implantations were performed by one of two anaesthesiologists (Dr W-Y Lin and Dr C-P Lin) who specialised in this procedure.

Endpoints. The primary endpoint of this study was the occurrence of catheter-related thrombotic or occlusion events, which included catheter-related venous thrombosis and intraluminal thrombosis. Catheter-related venous thrombosis was diagnosed through vascular duplex ultrasound (non-compressibility of the vein) or computed tomography as routine clinical practice at NTUH when patients experienced symptoms such as swelling and pain of the arm. Intraluminal thrombosis was defined as failure of infusion or failure to withdraw blood from TIVADs. Secondary endpoints included time to the occurrence of catheter-related thrombotic events, catheter-related venous thrombosis, intraluminal thrombosis, and catheter-related infection.

Catheter-related infection included catheter-related bloodstream infection (CRBSI) and catheter colonisation. CRBSI was defined as culture of the same organism from both the catheter segment and at least one blood culture from a peripheral vein or culture of the same organisms from at least two blood samples, one from the peripheral vein and the other from TIVAD, with the latter to be reported as positive at least $2 \mathrm{~h}$ sooner than the former (Chatzinikolaou et al, 2004). Catheter colonisation was defined as $>15$ colony-forming units in culture of the catheter segment or positive blood culture via TIVAD with pathogens typically causing catheter-related infection (Staphylococcus epidermidis, Staphylococcus aureus, and Candida species) (Hentrich et al, 2014).

Follow-up. We combined regular telephone contacts, patient reports, and medical record reviews for follow-up. Telephone contacts were performed every 4 weeks with a medical record review in the meantime to determine the aforementioned endpoints. Follow-up was continued until catheter removal or death.

Maintenance care for all catheters was provided by nurses in the chemotherapy room and oncology-related wards who have received adequate training. No blood withdrawal from TIVADs was permitted unless there was need for blood culture. All TIVADs were flushed with heparin solution $100 \mathrm{U} \mathrm{ml}^{-1}$ after every use or every 6 weeks if they were not utilised for a prolonged period.

Statistical analysis. The patient number was estimated based on the hypothesised thrombotic or occlusion event rates $(10 \%$ for right-sided implantation and 15\% for left-sided implantation), with $\alpha=0.05$ and $\beta=0.20$ under $1: 1$ randomisation. The loss to follow-up rate was expected to be $10 \%$. A total of 246 patients were required. The Kaplan-Meier method was used to estimate survival, and the log-rank test was used in a univariate analysis to compare survival between groups. To estimate the hazard ratios of factors predicting the occurrence of endpoints in univariate and multivariate analyses, a Cox proportional hazards model was used. All statistical analyses were performed using the SAS statistical software (Version 9.4, The SAS Institute, Cary, NC, USA). A two-sided $P$-value of $\leqslant 0.05$ was considered statistically significant.

\section{RESULTS}

Patient characteristics. Between 27 March 2012 and 30 March 2015, 246 patients signed informed consent. Of these, six patients withdrew before randomisation, and another five patients withdrew after randomisation but before undergoing the surgical 


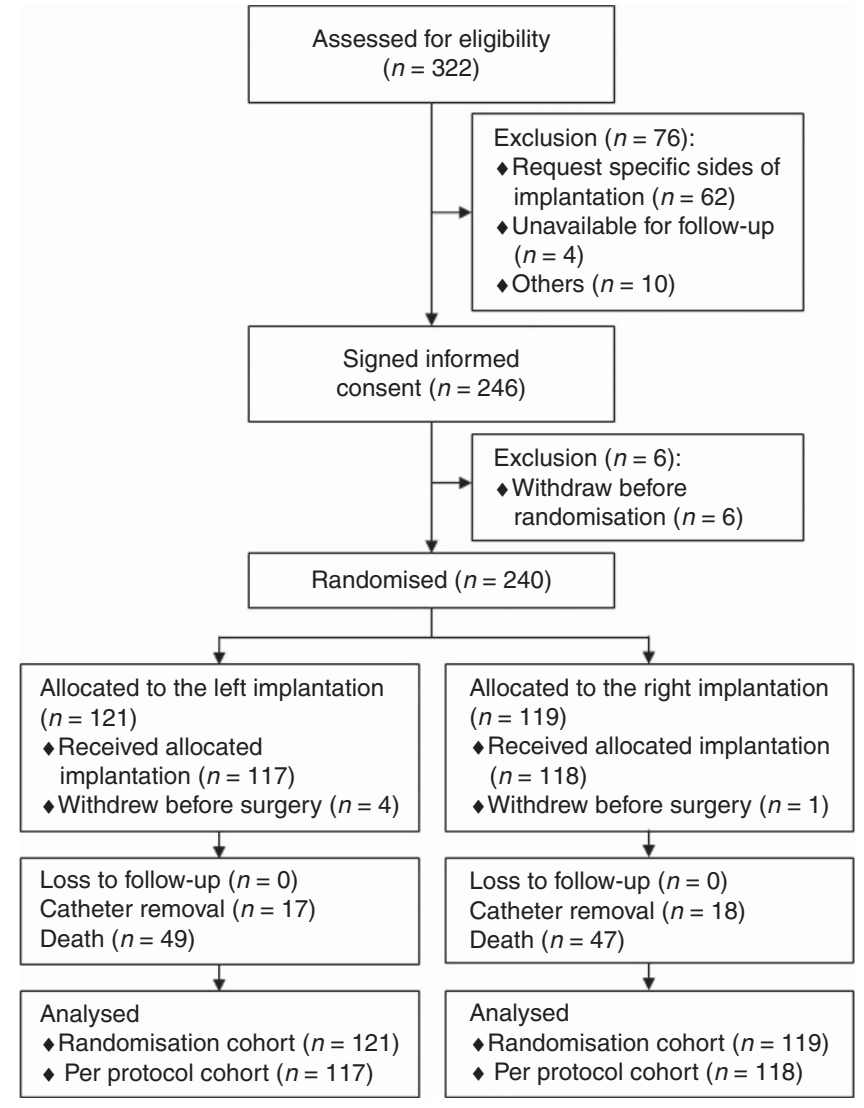

Figure 1. Patient flow diagram.

procedure (Figure 1). Finally, 235 patients received the TIVAD implantation according to the protocol.

The characteristics of patients in the randomised patient cohort $(n=240)$ and the per-protocol cohort $(n=235)$ are listed in Table 1. All patients received TIVAD implantation for chemotherapy administration. Demographic data were similar between the two cohorts. In the per-protocol cohort, $47 \%$ of the patients were female, and the most common cancer type was colorectal cancer (33\%), followed by lung (22\%), pancreatic (9\%), and gastric (9\%) cancer. Furthermore, $34 \%$ and $44 \%$ of the patients had stage III and stage IV cancers, respectively, upon enrolment. The mean age of the patients was 60.7 years. Before the implantation, five patients regularly received antiplatetlet agents; however, no patients received regular anticoagulation. Venous puncture was successful at the first attempt in most $(87 \%)$ patients; the mean number of venous puncture attempts was 1.3. No accidental arterial puncture occurred during venous puncture.

After randomisation, 117 and 118 patients received TIVAD implantation on the left and right sides, respectively. The patients who received implantation on different sides generally had similar demographic data. However, the men $(P=0.042)$ and the patients with lymphoma $(P=0.044)$ were more likely to receive left-sided implantation (Table 1). Left-sided implantation was associated with a higher possibility of the catheter tip being placed at the right atrium-superior vena cava junction $(P=0.001$; Table 1$)$.

Patient outcomes. By the last follow-up date of 31 March 2016, the median follow-up time was 24.8 months; 73 (62\%) patients with left-sided TIVAD implantation and 73 (62\%) patients with right-sided TIVAD implantation had discontinued the study. The most common causes were patient death and catheter removal. The median time to catheter removal or death was 22.8 months $(95 \%$ confidence interval, 17.7-27.9).
Catheter-related thrombotic or occlusion events occurred in 9 (4\%) patients, accounting for 0.065 events per 1000 catheter-days. The occurrence rates were similar between the patients with left- and right-sided implantations (5 vs 3\%, $P=0.333$; Table 2). When we compared time from catheter implantation to the occurrence of these thrombotic or occlusion events, the results between the two groups remained similar $(P=0.328$, Figure $2 \mathrm{~A})$. The 24 -month thrombosis or occlusion rate was $5.7 \%$ and $3.6 \%$ in the patients with left- and right-sided catheter implantation, respectively.

Catheter-related venous thrombosis occurred in only $2(1 \%)$ patients, accounting for 0.014 events per 1000 catheter days. These events occurred in one patient with left-sided implantation and one patient with right-sided implantation, so the side of implantation had no significant associations with the occurrence of vessel thrombosis $(P=1.000)$. Both venous thrombosis events improved after anticoagulation, and the TIVADs remained functional. Intraluminal thrombosis occurred in 7 (3\%) patients; all of these events were reversed using urokinase or heparin.

Catheter-related infection was observed in 26 (11\%) patients (Table 2), accounting for 5.7 events per 1000 catheter-days. The occurrence rate was similar between the patients with left- and right-sided implantations ( 9 vs $14 \%, P=0.221$ ), as was the time from catheter implantation to the occurrence of catheter-related infection $(P=0.222$, Figure $2 \mathrm{~B})$. The 12 -month catheter-related infection rate was $7.6 \%$, and $9.7 \%$ in the patients with left- and right-sided implantations, respectively; the 24-month infection rate was $10.7 \%$ and $14.8 \%$, respectively. CRBSI and catheter colonisation occurred in $9 \%$ and $5 \%$ of the patients, respectively.

Among patients with catheter-related infection, the median time to infection was 8.3 months (Supplementary Figure S1). The most commonly identified pathogens included Staphylococcus epidermidis $(n=4)$ and Staphylococcus aureus $(n=3)$ (Supplementary Table S1). Gram-positive bacteria, gram-negative bacteria, and Candida species were identified in 11 (42\%), 10 (38\%), and 5 (19\%) patients, respectively.

We examined several potential factors associated with catheterrelated thrombosis. Age, sex, cancer diagnosis, disease stage, previous mediastinal radiotherapy, tip location, handedness, number of venous puncture attempts, operator, body mass index, and performance status, were analysed first in a univariate and then in a multivariate analysis (Table 3). Platelet count, prothrombin time, activated partial thrombin time (APTT), D-dimer level, and fibrinogen level before surgery were also included. The side of the implantation remained unassociated with catheter-related thrombosis in the multivariate analysis. Moreover, longer APTT was associated with less catheter-related thrombosis (adjusted hazard ratio, 0.653; $P=0.050$ ).

\section{DISCUSSION}

In this prospective randomised observation study, we observed that the side of central line TIVAD implantation was not a predictive factor for catheter-related thrombotic events or infection. Many potential confounding factors, such as catheter types and operators were well controlled. Most other confounding factors were equally distributed in the two randomised groups. Although the unexpected low incidence of catheter-related thrombotic or occlusion events reduced the statistical power of the study, this is the first prospective randomised study to address this issue.

The low incidence of catheter-related thrombotic or occlusion events in this study could be attributed to multiple factors. Almost $40 \%$ of patients still had TIVAD in place, so the incidence will further increase. We excluded patients with a very high possibility of catheter-related thrombotic events, such as patients with large 
Table 1. Patient characteristics of the randomisation cohort and the per protocol cohort

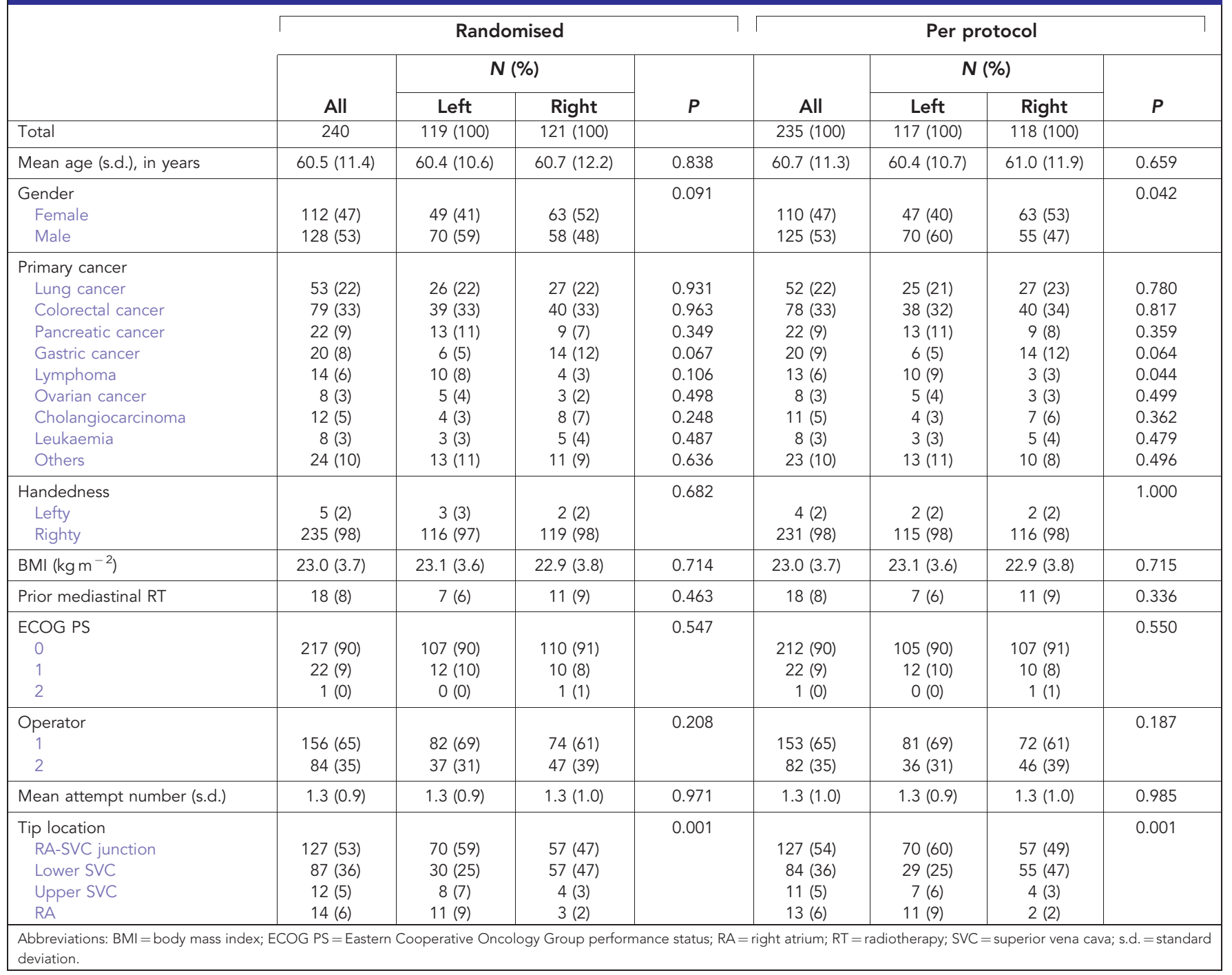

\begin{tabular}{|c|c|c|c|c|}
\hline$N(\%)$ & All $(N=235)$ & Left $(N=117)$ & Right $(N=118)$ & $P$ \\
\hline $\begin{array}{l}\text { Thrombotic or occlusion events } \\
\text { Venous thrombosis } \\
\text { Intraluminal thrombosis }\end{array}$ & $\begin{array}{l}9(4) \\
2(1) \\
7(3)\end{array}$ & $\begin{array}{l}6(5) \\
1(1) \\
5(4)\end{array}$ & $\begin{array}{l}3(3) \\
1(1) \\
2(2)\end{array}$ & $\begin{array}{l}0.333 \\
1.000 \\
0.281\end{array}$ \\
\hline $\begin{array}{l}\text { Catheter-related infection } \\
\text { CRBSI } \\
\text { Catheter colonisation }\end{array}$ & $\begin{array}{l}26(11) \\
20(9) \\
12(5)\end{array}$ & $\begin{aligned} & 10(9) \\
& 7(6) \\
& 5(4)\end{aligned}$ & $\begin{array}{l}16(14) \\
13(11) \\
7(6)\end{array}$ & $\begin{array}{l}0.221 \\
0.167 \\
0.564\end{array}$ \\
\hline
\end{tabular}

mediastinal tumours. We did not perform active surveillance (i.e., regular Doppler ultrasound examinations); thus, all catheterrelated venous thrombosis events in this study were symptomatic. However, the prevalence of such symptomatic venous thrombi remained much lower than that reported in Western countries, which was approximately 9-12\% (Kuter, 2004; Beckers et al, 2010). All of the patients in this study were of Asian ethnicity, which may be associated with a lower chance of venous thromboembolism (Klatsky et al, 2000; Lee et al, 2006; Oh et al, 2008).

Both of the operators who performed the implantation in this study were well experienced in performing this surgical procedure.
The number of attempts in venous puncture, which was reported as a risk factor for catheter-related thrombotic events (Lee et al, 2006; Murray et al, 2013), was generally low in our study; this was probably due to the selection of ultrasound-assisted subclavian insertion (Biffi et al, 2009). Almost 90\% of the patients required only one venous puncture, which reduced the endothelial damage. All of the patients in this study received implantation of catheters made of polyurethane, which may also reduce thrombosis compared with catheters made of polyvinylchloride, tetrafluoroethylene, or polyethylene (Linder et al, 1984; Gallieni et al, 2008; Shivakumar et al, 2009). 

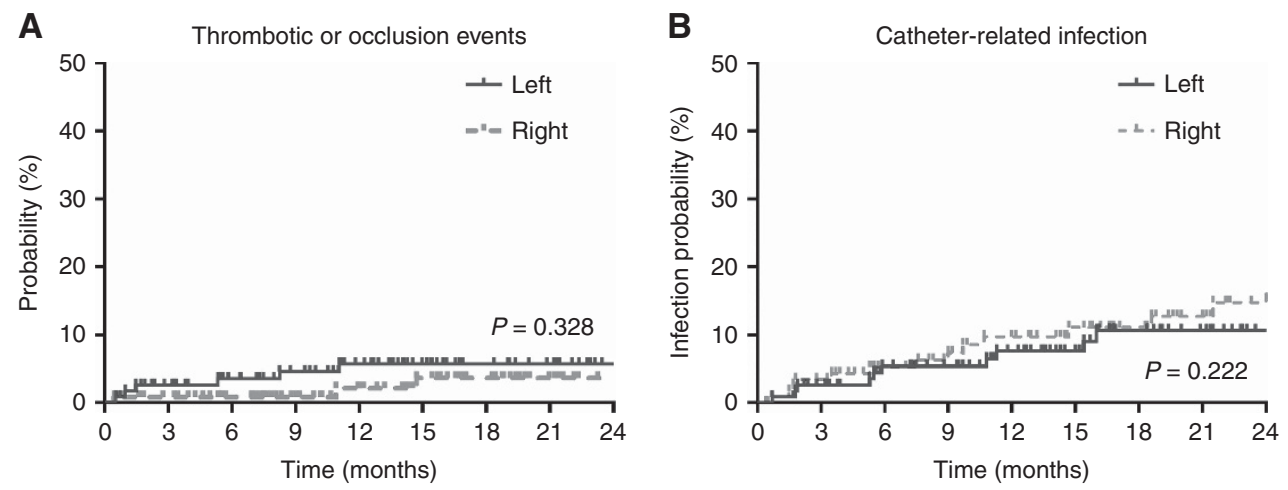

Figure 2. Time to (A) all catheter-related thrombotic or occlusion events and (B) catheter-related infection. Patients are grouped by the side of the TIVAD implantation. P-values are conducted using the log-rank test.

Table 3. Univariate and multivariate analysis of potential predictive factors of thrombotic events by a Cox proportional hazards model

\begin{tabular}{|c|c|c|c|c|c|c|}
\hline Covariates & \multicolumn{3}{|c|}{ Univariate } & \multicolumn{3}{|c|}{ Multivariate } \\
\hline Side of implantation & 1.939 & $(0.492-7.873)$ & 0.338 & 2.895 & $(0.009-885.722)$ & 0.716 \\
\hline Age & 1.010 & $(0.953-1.071)$ & 0.733 & 1.014 & (0.938-1.096) & 0.732 \\
\hline Diagnosis & 0.948 & $(0.721-1.246)$ & 0.701 & 0.970 & $(0.685-1.373)$ & 0.863 \\
\hline Side of implantation & 1.939 & $(0.492-7.873)$ & 0.338 & 2.895 & $(0.009-885.722)$ & 0.716 \\
\hline Tip location & 0.356 & $(0.087-1.453)$ & 0.150 & 0.472 & $(0.116-1.918)$ & 0.294 \\
\hline Implant side $\times$ handedness & 0.513 & $(0.128-2.053)$ & 0.346 & 0.913 & $(0.003-280.384)$ & 0.975 \\
\hline Attempt number & 0.694 & $(0.170-2.832)$ & 0.611 & 0.584 & $(0.107-3.195)$ & 0.535 \\
\hline Operator & 0.909 & $(0.227-3.635)$ & 0.892 & 0.670 & $(0.133-3.382)$ & 0.627 \\
\hline Prothrombin time $^{a}$ & 0.614 & $(0.176-2.137)$ & 0.443 & 1.477 & $(0.277-7.873)$ & 0.648 \\
\hline $\mathrm{APTT}^{\mathrm{a}}$ & 0.717 & $(0.538-0.954)$ & 0.023 & 0.653 & $(0.427-0.999)$ & 0.050 \\
\hline D-dimer ${ }^{a}$ & 0.993 & $(0.874-1.128)$ & 0.912 & 0.941 & $(0.810-1.094)$ & 0.429 \\
\hline Fibrinogen $^{a}$ & 0.997 & (0.990-1.003) & 0.315 & 0.998 & $(0.989-1.006)$ & 0.581 \\
\hline
\end{tabular}

We found that the patients who received TIVAD implantation on the left side were more likely to have the catheter tip at the optimal location, which was the right atrium-superior vena cava junction (Caers et al, 2004; Kuter, 2004; Verso et al, 2008). Gender distribution was imbalanced in the per-protocol cohort, and the male patients and patients with lymphoma were more likely to receive TIVAD implantation on the left side. However, after including the tip location, gender, and cancer diagnosis as covariates in the multivariate analysis, the side of the implantation was still not identified as a predictive factor for catheter-related thrombotic or occlusion events.

The incidence of catheter-related infection or CRBSI was slightly higher than that reported in the literature (Eastman et al, 2001; Chen et al, 2013; Freire et al, 2013; Wang et al, 2015). In our study, more than $40 \%$ of the patients had stage IV disease; $9 \%$ of the patients had haematological disease and another $9 \%$ of the patients had pancreatic cancer. These factors may have contributed to the higher incidence of catheter-related infection (Toure et al, 2012; Lipitz-Snyderman et al, 2014; Wang et al, 2015).

In conclusion, we demonstrated that the side of central line TIVAD implantation was not associated with the occurrence of catheter-related thrombotic or occlusion events in patients with cancer.

\section{ACKNOWLEDGEMENTS}

We thank Prof Po-Ren Hsueh from National Taiwan University Hospital, Taipei, Taiwan for his help with the summary of catheter-related infection events. This study was supported by grants from the Ministry of Science and Technology, Taiwan (MOST-102-2314-B-002-120, MOST-103-2314-B-002-181-MY2, and MOST-105-2314-B-002-194) and National Taiwan University Hospital (NCTRC201208, NTUH. 103-S2314, NTUH. 105S2954, and NCTRC201603). 


\section{CONFLICT OF INTEREST}

The authors declare no conflict of interest.

\section{REFERENCES}

Anderson AJ, Krasnow SH, Boyer MW, Cutler DJ, Jones BD, Citron ML, Ortega LG, Cohen MH (1989) Thrombosis: the major Hickman catheter complication in patients with solid tumor. Chest 95: 71-75.

Barzaghi A, Dell'Orto M, Rovelli A, Rizzari C, Colombini A, Uderzo C (1995) Central venous catheter clots: incidence, clinical significance and catheter care in patients with hematologic malignancies. Pediatr Hematol Oncol 12: 243-250.

Beckers MM, Ruven HJ, Seldenrijk CA, Prins MH, Biesma DH (2010) Risk of thrombosis and infections of central venous catheters and totally implanted access ports in patients treated for cancer. Thromb Res 125: 318-321.

Biffi R, Orsi F, Pozzi S, Pace U, Bonomo G, Monfardini L, Della Vigna P, Rotmensz N, Radice D, Zampino MG, Fazio N, de Braud F, Andreoni B, Goldhirsch A (2009) Best choice of central venous insertion site for the prevention of catheter-related complications in adult patients who need cancer therapy: a randomized trial. Ann Oncol 20: 935-940.

Caers J, Fontaine C, Vinh-Hung V, Mey J, Ponnet G, Oost C, Lamote J, Greve J, Camp B, Lacor P (2004) Catheter tip position as a risk factor for thrombosis associated with the use of subcutaneous infusion ports. Support Care Cancer 13: 325-331.

Chatzinikolaou I, Hanna H, Hachem R, Alakech B, Tarrand J, Raad I (2004) Differential quantitative blood cultures for the diagnosis of catheterrelated bloodstream infections associated with short- and long-term catheters: a prospective study. Diagn Microbiol Infect Dis 50: 167-172.

Chen IC, Hsu C, Chen YC, Chien SF, Kao HF, Chang SY, Hu FC, Yeh KH (2013) Predictors of bloodstream infection associated with permanently implantable venous port in solid cancer patients. Ann Oncol 24: 463-468.

Craft PS, May J, Dorigo A, Hoy C, Plant A (1996) Hickman catheters: leftsided insertion, male gender, and obesity are associated with an increased risk of complications. Aust NZ J Med 26: 33-39.

De Cicco M, Matovic M, Balestreri L, Panarello G, Fantin D, Morassut S, Testa V (1997) Central venous thrombosis: an early and frequent complication in cancer patients bearing long-term silastic catheter. A prospective study. Thromb Res 86: 101-113.

Doizaki KK, Weber JN (1986) Restoring patency to occluded venous access ports. Am J Hosp Pharm 43: 880.

Eastman ME, Khorsand M, Maki DG, Williams EC, Kim K, Sondel PM, Schiller JH, Albertini MR (2001) Central venous device-related infection and thrombosis in patients treated with moderate dose continuousinfusion interleukin-2. Cancer 91: 806-814.

Eastridge BJ, Lefor AT (1995) Complications of indwelling venous access devices in cancer patients. J Clin Oncol 13: 233-238.

Fraschini G, Jadeja J, Lawson M, Holmes FA, Carrasco HC, Wallace S (1987) Local infusion of urokinase for the lysis of thrombosis associated with permanent central venous catheters in cancer patients. J Clin Oncol 5: 672-678.

Freire MP, Pierrotti LC, Zerati AE, Araujo PH, Motta-Leal-Filho JM, Duarte LP, Ibrahim KY, Souza AA, Diz MP, Pereira J, Hoff PM, Abdala E (2013) Infection related to implantable central venous access devices in cancer patients: epidemiology and risk factors. Infect Control Hosp Epidemiol 34: 671-677.

Gallieni M, Pittiruti M, Biffi R (2008) Vascular access in oncology patients. CA Cancer J Clin 58: 323-346.

Gould JR, Carloss HW, Skinner WL (1993) Groshong catheter-associated subclavian venous thrombosis. Am J Med 95: 419-423.

Hentrich M, Schalk E, Schmidt-Hieber M, Chaberny I, Mousset S, Buchheidt D, Ruhnke M, Penack O, Salwender H, Wolf HH, Christopeit M, Neumann S, Maschmeyer G, Karthaus M, Infectious
Diseases Working Party of the German Society of H, Medical O (2014) Central venous catheter-related infections in hematology and oncology: 2012 updated guidelines on diagnosis, management and prevention by the Infectious Diseases Working Party of the German Society of Hematology and Medical Oncology. Ann Oncol 25: 936-947.

Hurtubise MR, Bottino JC, Lawson M, McCredie KB (1980) Restoring patency of occluded central venous catheters. Arch Surg 115: 212-213.

Klatsky AL, Armstrong MA, Poggi J (2000) Risk of pulmonary embolism and/ or deep venous thrombosis in Asian-Americans. Am J Cardiol 85: 1334-1337.

Kuter DJ (2004) Thrombotic complications of central venous catheters in cancer patients. Oncologist 9: 207-216.

Lee AY, Levine MN, Butler G, Webb C, Costantini L, Gu C, Julian JA (2006) Incidence, risk factors, and outcomes of catheter-related thrombosis in adult patients with cancer. J Clin Oncol 24: 1404-1408.

Lin CP, Wang YC, Lin FS, Huang CH, Sun WZ (2011) Ultrasound-assisted percutaneous catheterization of the axillary vein for totally implantable venous access device. Eur J Surg Oncol 37: 448-451.

Linder LE, Curelaru I, Gustavsson B, Hansson HA, Stenqvist O, Wojciechowski J (1984) Material thrombogenicity in central venous catheterization: a comparison between soft, antebrachial catheters of silicone elastomer and polyurethane. JPEN J Parenter Enteral Nutr 8 : 399-406.

Lipitz-Snyderman A, Sepkowitz KA, Elkin EB, Pinheiro LC, Sima CS, Son CH, Atoria CL, Bach PB (2014) Long-term central venous catheter use and risk of infection in older adults with cancer. J Clin Oncol 32: 2351-2356.

Murray J, Precious E, Alikhan R (2013) Catheter-related thrombosis in cancer patients. Br J Haematol 162: 748-757.

Nightingale CE, Norman A, Cunningham D, Young J, Webb A, Filshie J (1997) A prospective analysis of 949 long-term central venous access catheters for ambulatory chemotherapy in patients with gastrointestinal malignancy. Eur J Cancer 33: 398-403.

Oh SY, Kim JH, Lee KW, Bang SM, Hwang JH, Oh D, Lee JS (2008) Venous thromboembolism in patients with pancreatic adenocarcinoma: lower incidence in Asian ethnicity. Thromb Res 122: 485-490.

Press OW, Ramsey PG, Larson EB, Fefer A, Hickman RO (1984) Hickman catheter infections in patients with malignancies. Medicine (Baltimore) 63 : 189-200.

Ray S, Stacey R, Imrie M, Filshie J (1996) A review of 560 Hickman catheter insertions. Anaesthesia 51: 981-985.

Schwarz RE, Coit DG, Groeger JS (2000) Transcutaneously tunneled central venous lines in cancer patients: an analysis of device-related morbidity factors based on prospective data collection. Ann Surg Oncol 7: 441-449.

Shivakumar SP, Anderson DR, Couban S (2009) Catheter-associated thrombosis in patients with malignancy. J Clin Oncol 27: 4858-4864.

Stanislav GV, Fitzgibbons Jr RJ, Bailey Jr RT, Mailliard JA, Johnson PS, Feole JB (1987) Reliability of implantable central venous access devices in patients with cancer. Arch Surg 122: 1280-1283.

Toure A, Vanhems P, Lombard-Bohas C, Cassier P, Pere-Verge D, Souquet JC, Ecochard R, Chambrier C (2012) Totally implantable central venous access port infections in patients with digestive cancer: incidence and risk factors. Am J Infect Control 40: 935-939.

Verso M, Agnelli G, Kamphuisen PW, Ageno W, Bazzan M, Lazzaro A, Paoletti F, Paciaroni M, Mosca S, Bertoglio S (2008) Risk factors for upper limb deep vein thrombosis associated with the use of central vein catheter in cancer patients. Intern Emerg Med 3: 117-122.

Wang TY, Lee KD, Chen PT, Chen MC, Chen YY, Huang CE, Kuan FC, Chen CC, $\mathrm{Lu} \mathrm{CH}$ (2015) Incidence and risk factors for central venous access port-related infection in Chinese cancer patients. J Formos Med Assoc 114: 1055-1060.

This work is published under the standard license to publish agreement. After 12 months the work will become freely available and the license terms will switch to a Creative Commons AttributionNonCommercial-Share Alike 4.0 Unported License.

Supplementary Information accompanies this paper on British Journal of Cancer website (http://www.nature.com/bjc) 